\title{
Active Control of Radiated Sound from Stiffened Plates Using IDE-PFC Actuators
}

\author{
Atanu Sahu \\ Institute of Composite Structures and Adaptive Systems, DLR, 38108 Braunschweig, Germany \\ Tirtha Banerjee, Arup Guha Niyogi and Partha Bhattacharya \\ Department of Civil Engineering, Jadavpur University, Kolkata -700032, India
}

(Received 29 November 2011; revised 7 January 2013; accepted 6 March 2013)

It has been a practice in modern day aircraft and automobile industries to manufacture a stiffened structure for significantly enhancing efficiency and strength without incurring a considerable weight increase. In the present study, an attempt is made to understand the effect of stiffeners on the sound radiation pattern of a vibrating plate. Subsequently, a velocity feedback control algorithm based on Linear Quadratic Regulator (LQR) methodology is developed to attenuate the radiated sound power from the vibrating structures with surface bonded piezo fibre composite (PFC) patches with interdigitated electrodes (IDE) as actuators and polyvinylidene fluoride (PVDF) films as sensors. Results are obtained for different orientations of stiffeners and various locations of PFC patches and are discussed.

\section{NOMENCLATURE}

$\begin{array}{ll}\omega & \text { Natural frequency } \\ \hat{\sigma} & \text { Radiation efficiency } \\ \{\sigma\} & \text { Stress vector } \\ \{\varepsilon\} & \text { Strain vector } \\ \{\mathrm{E}\} & \text { Electric field vector } \\ \mathrm{E} & \text { Young's modulus } \\ \nu & \text { Poisson's ratio } \\ \rho & \text { Material density } \\ {[\mathrm{Q}]} & \text { Elastic moduli matrix } \\ \{\mathrm{D}\} & \text { Electrical displacement vector } \\ {[\mathrm{e}]} & \text { Piezoelectric stress/charge constant } \\ {[x]} & \text { Electric permittivity or dielectric matrix } \\ {[\mathrm{p}]} & \text { Inertia matrix } \\ {[\mathrm{N}]} & \text { Shape function matrix } \\ \{d\},\{\dot{d}\},\{\ddot{d}\} & \text { Generalized displacement, velocity, and } \\ {[\mathrm{M}]} & \text { acceleration vector } \\ {\left[\mathrm{K}_{\mathrm{UU}}\right]} & \text { Mass matrix } \\ {\left[\mathrm{K}_{u \phi}\right]} & \text { Mechanical stiffness matrix } \\ {[\mathrm{K}} & \text { Electro-mechanical coupling stiffness } \\ {[\mathrm{Z}]} & \text { matrix } \\ {[\mathrm{B}]} & \text { Electrical stiffness matrix } \\ \hat{Q} & \text { Position matrix of the piezo patch } \\ \left\{\mathrm{F}_{\mathrm{el}}\right\} & \text { Strain-displacement matrix } \\ \{y\},\{\dot{y}\},\{\ddot{y}\} & \text { Electrical charge } \\ & \text { Electrical load vector } \\ & \text { Displacement, velocity, and acceleration } \\ & \text { vector in modal level } \\ & \end{array}$

\section{INTRODUCTION}

In the present day automobile and aerospace industry, the objective is to increase fuel efficiency without compromising structural stiffness and strength. To achieve these objectives, the present trend is to use lightweight structural elements.
However, these forms of structures have the possibility of generating unacceptable levels of sound and noise due to vibration. Since interior noise has a strong effect on passenger comfort and acceptance of the vehicle, researchers and engineers are continually working on different methodologies to reduce the noise level.

Classically, sound attenuation in the medium to high frequency acoustic range can be achieved in a passive manner by adding sound absorbing materials to the surface of the radiating structure. An alternate methodology is to use destructive interference in the sound source path. A more advanced form of the sound control is based on altering the vibrations of the noisy structure such that it radiates less sound. This alteration may be achieved by introducing discrete and collocated sensors and actuator pairs that fall under the scope of active control strategy.

The acoustic radiation problem has been addressed by various researchers, including Gladwell, ${ }^{1}$ Gladwell and Zimmerman, ${ }^{2}$ Seybert et al., ${ }^{3}$ and others. Cunefare has shown that the radiated sound power can be expressed in terms of acoustic radiation filters, surface radiation modes, and discrete surface velocities of the vibrating structure. ${ }^{4}$ Elliot and Johnson compared two different formulations for calculating the acoustic power radiated from a vibrating structure in the free field and then implemented a feed forward control of sound power for a baffled square panel. ${ }^{5}$ Radiation efficiencies and singular velocity patterns were introduced by Borgiotti and Jones using the singular value decomposition (SVD) of a radiation resistance matrix. ${ }^{6}$ Gibbs et al. developed a radiation modal expansion (RME) technique to reduce computational effort and then apply a feedback control strategy with radiation filters employing multiple input or output smart sensor-actuators. ${ }^{7}$ Bhattacharya et al. have shown that the size and geometry of the vibrating structure influences the cut-off limit of the acoustic ra- 